\title{
NOTE SUR LA FECONDATION ARTIFICIELLE ET LE DEVELOPPEMENT DES ALEVINS DE PERCHE
}

\author{
par J. ARLET
}

Assistante la Slation centrale d'Hydrobiologie appliquée.

Ces observations ont été faites à la Station d'Hydrobiologie du Paraclet, où l'année dernière $\mathrm{A}$. Wuntz avait procédé à une étude intéressante sur les jeunes alevins de Brochet.

Il est probable que ni la fécondation artificielle des Perches, ni leur élevage en bacs n'ont été effectués en France, et dans la Littérature étrangère, il n'est pas fait mention, à ma connaissance, qu'un essai de ce genre ait élé tenté.

O. Schinder a étudié des alevins de Perche de tous áges, mais ils provenaient d'un étang où la reproduction et leur développement avaient lieu dans des conditions naturelles.

L'obtention et l'élevage des jeunes Perches s'est révélé difficile et je n'ai pu conserver les alevins en aquarium plus de dix jours.

Les difficultés commencent dès l'opération de ponte et de fécondation artificielles.

Les pontes de Perche se présentent sous l'aspect de rubans muqueux que I'on trouvęęn Avril accrochés aux herbes des étangs; longs de 40 centimètres, larges de 5 centimètres, ils ont l'aspect d'un réseau dont les oulis forment les mailles ; collés les uns aux autres, ce qui leur donne une forme presque polygonale, les cufs, de couleur blanche, mesurent $2 \mathrm{~mm}$. i) de diamètre.

Il fallut plusieurs essais avant qu'une femelle laissat sortir ses oufs. L'une d'elie, présumée mûrc, ne consentit pas à pondre sous la pression de la main ; le lendemain, quoique séparée des mâles, elle avait frayé dans le bac. Ce qui empêche une ponte facile, c'est que les oufs se trouvent déjà agglutinés en rubans dans le ventre de la femelle; il faut donc que les femelles soient tout à fait mûres pour qu'une légère pression suffise : l'expulsion des œufs. 
La técondation a été effectuée par la méthode sèche en mélangeant intimement oufs et laitance avec une plume. Lagencement des œufs en cordon rend très difficile un mélange parfait et il est impossible que tous les ovules soient en contact avec les spermatozoïdes ; en fait, seulement $50 \%$ sont fécondés. Nombre d'entre eux meurent, et restant accolés aux cuf́s fécondés, contaminent ceux-ci sans que l'on puisse les enlever.

L'emploi de la bouteille de Zug pour l'incubation est aussi très malcommode ; le ruban d'oufs risque continuellement de s'échapper par l'ouverture de la bouteille.

Les premiers essais d'incubation ont été faits dans les bacs à Truites de la.Pisciculture. La température de l'eau était environ de $12^{\circ}$; elle s'est révélée trop froide et les oufs sont morts avant l'éclosion. D'autres en provenance d'une ponte d'étang ont été placés également dans les bacs. Les alevins remuaient déjà à l'intérieur des cufs ; le changement d'eau, ou plutôt l'abaissement de température les a tués avant leur éclosion.

Les ceufs fécondés ont alors été mis en incubation dans des aquariums de laboratoire où l'eau était à $13^{\circ}$. L'incubation nécessite seulement huit jours ; on sait qu'en multipliant la température de l'eau par le nombre de jours, on obtient un chiffre classique appelé "somme totale de degrés nécessaires pour l'éclosion ", qui est ici de $104^{\circ}$. D'après K. Smolian, le développement de l'oeuf exigerait $80^{\circ}$, d'après F. Dusprya, 98 ; O. ScminDLFn, lui, a observé l'éclosion seulement pour une somme de 140 à $160^{\circ}$, ce qui m'apparałt un peu trop élevé.

La période d'incubation des Perches est assez courte par rapport aux autres espèces de Poissons ; la Carpe nécessite $114^{\circ}$ au moins, le Brochet 120 à $131^{\circ}, 1^{\prime}$ Ombre environ $200^{\circ}$, la Truite 300 a u moins.

Dès le $5^{\circ}$ jour d'incubation, l'alevin commence à remuer dans l'œuf et ses deux gros yeux sont nettement visibles, noirs et brillants ; à l'éclosion, l'alevin est remarquable par sa transparence ; au $10^{\circ}$ jour il est encore transparent ; c'est déjà un caractère qui permet de le distinguer de la plupart des autres alevins. Mais son comportement dans l'aquarium le caractérise encore davantage.

Au sortir de l'œuf, l'alevin ne reste pas inactif ; il commence à nager vivement, en se tenant le plus souvent en position verticale; il monte d'abord perpendiculairement à la surface de l'eau, puis se laisse retomber, pour recommencer son ascension aussitôt ; il ne se repose jamais sur le fond de l'aquarium et est continuellement en mouvement; ce manège dure pendant 4 ou 5 jours.

$\mathrm{Au} 5^{\circ}$ jour, l'alevin prend une position horizontale, mais il continue à nager activement et ne se tient pas immobile sur le fond ou sous la surface de l'eau comme le font les alevins de Brochet. 


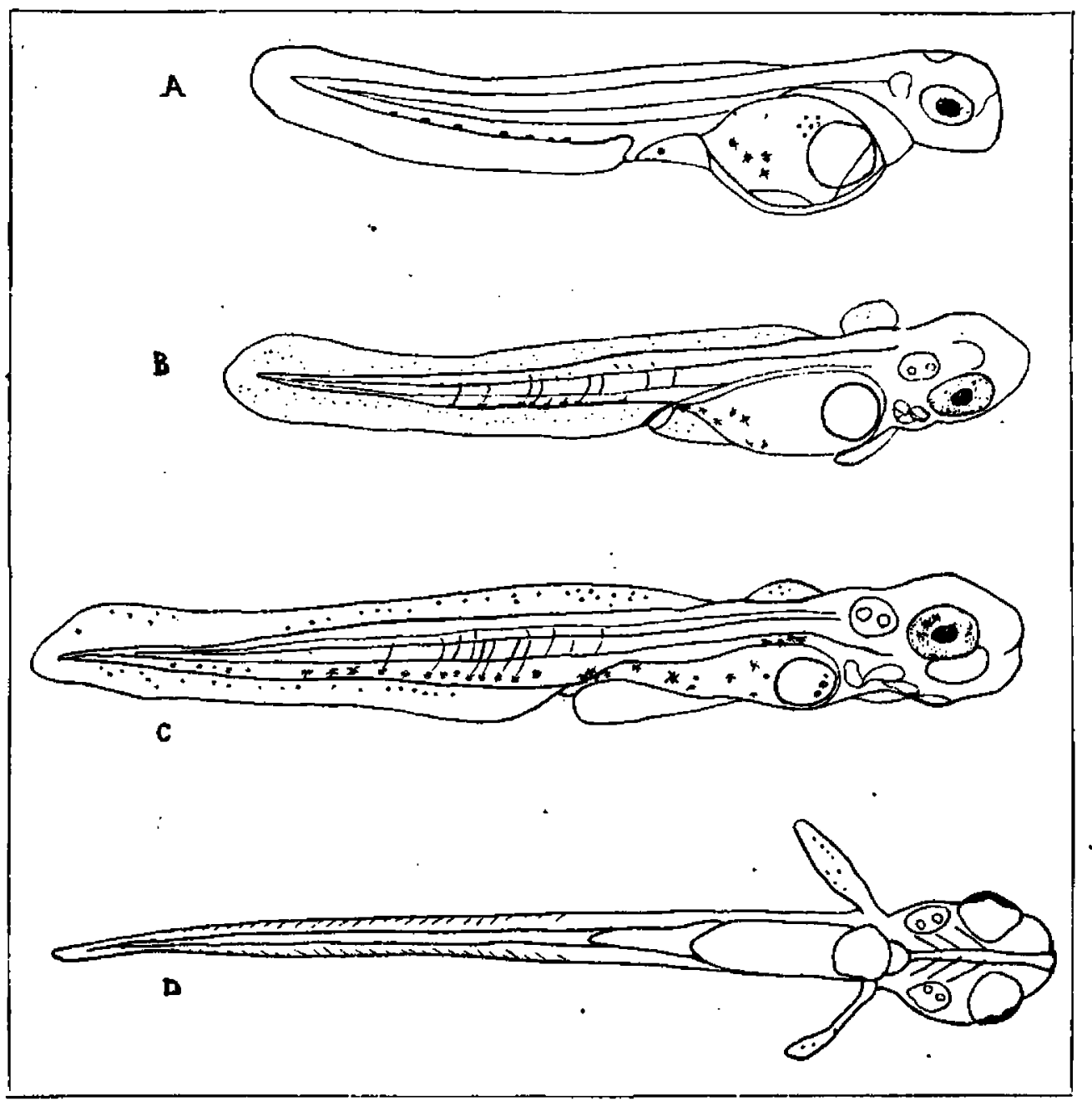

Fig. I. - Alevins do.Perches.

Frg. A, l'éclosion; - Fig. B, Alevin de 3 jours; - Fig. C, Alevin de 8 jours; - Fig. D, le méme en coupe ventrale.

\section{Description de l'alevin à l'éclosion.}

Il est transparent et très petit : sa longueur totale est environ 4,8 à 5 millimètres ; un jeune Brochet, à l'éclosion, mesure environ 5 millimètres, une Carpe 4,5 à 5 millimètres, une Truite 10 à 15 millimètres.

La tête est légèrement infléchie sur la vésicule vitelline; les yeux très gros sont irisés et brillants. La bouche n'est pas encore fendue. La vésicule vitelline peu volumineuse et transparente a une forme ovoïde. Dans sa partie antérieure, se trouve un seul globule graisseux de grande taille, parfaitement opaque.

Les nageoires pectorales sont différenciées ; une seule nageoire embryon- 
naire ou proptérygie contourne le corps de l'alevin, commence à la pointe postérieure de la vésicule vitelline et se termine dorsalement en arrière des nageoires pectorales. Elle est interrompue dans la région abdominale au niveau de l'anus ou plutôt, car lintestin ne communique pas encorc avec l'extérieur, au niveau du tractus qui indique I'emplacement du futur anus ; celui-ci n'est pas situé au milieu du corps, mais bien en avant du corps et le rapport de la distance anus-museau d'une part, anuṣ-extrémité du corps de l'autre, est $11 / 15$.

La chorde devient visible en arrière des otocystes.

Le corps de lalevin est très peu pigmenté : quelques mélanophores étoilés sont disposés sur la parlie postérieure de la vésicule, et d'autres sont régulièrement espacés, suivant une ligne abdominale ; un ou deux mélanophores largement élalés se trouvent entre l'anus et l'extrémité de la vésicule.

Les dimensions de l'alevin à l'éclosion sont les suivantes :

Longucur totale : 5 millimètres ; - IIauteur de la tête : 0,5 millimètres; - OEil : $0,20 \times 0,25$ millimètre ; - Hauteur maxima du tronc : 0,95 millimètre; - Hauteur du tronc au niveau de l'anus : 0,3 millimètre; - Vésicule : 1,1 millimètre $\times 0,7$ millimètre; - Diamètre du grlobule graisseux : 0,5 millimètre ; - Longueur de la poinle du museau à l'anus : 2,3 millimètres ; - Longueur de l'anus à l'extrémité de la chorde : 2,6 millimètres ; - Rapport des deux longueurs : 0,88 .

\section{Alevins de 2 à 3 jours.}

Leur longueur est à peu près 5,5 à 5,6 millimètres. La tête n'est plus du tout infléchie et se tient dans le prolongcment du corps. La bouche n'est pas encore ouverte. Par transparence les deux otocystes contenant chacun deux granulations ou otolithes sont parfailement visibles.

Entre l'œil et la vésicule vitelline, on voit facilement battre le corur et mème les cavités se contracter successivement. Il n'est pas déplacé par la vésicule vitelline, comme cela se passé chez le Brochet et la Truite, chez lesquels la vésicule repousse le cour en une position verticale.

La vésicule vitelline s'est légèrement allongée ì sa partie antérieure, le globule graisseux a diminué un peu de volume. Les nageoires pectorales sont constamment étendues perpendiculairement au corps; elles sont parsemées de quelques mélanophores ponctués ; la proptérygie aussi porte de nombreux mélanophores, minuscules points nullement ordonnés.

Le corps de l'alevin est pigmenté davantage, tout en restant parfaitement transparent. La vésicule vitelline, dans sa partie postérieure, porte 6 à S mélanophores ramifiés, au voisinage de l'extrémité de l'jntestin ; quelques mélanophores forment une tache foncéc. $D$ 'autres, régulièrement disposés en une ligne bordent l'abdomen. Dans la région dorsale, une série 
de mélanophores paralleles indique les séparations entre les segments musculaires ou mỵotomes. La chorde est tout à fait droite, et non relevée à l'extrémité postérieure.

\section{Dimensions :}

Longueur totale : 5,5 millimêtres; - Hauteur de la tête : 0,67 millimètre ; - OEil : 0,25 x 0,3 millimètre ; - Hauteur maxima du tronc: 1, I millimètre; - Hauteur du tronc au niveau de l'anus : 0,36 millimètre; - Vésicule : r, $25 \times 0,5$ milljmètre ; - Globule : 0,3 millimètre de diamètre :Longueur du museau à l'anus : 2,5 millimètres; - Longueur de l'anus à l'extrémité de la chorde : 2,8 millimètres. - Rapport des deux longueurs : $\mathbf{0 , 8 9}$.

\section{Alevins de $\mathbf{5}$ à 6 jours.}

Leur longueur est environ de 5,8 millimètres. Peu de changement dans l'aspect du petit alevin. Il est encore transparent bien que la pigmentation soit un peu plus accentuée, en particulier dans la région anale. La bouche est fendue et presque en position terminale de la tête. Mais les mâchoires ne remuent pas encore à chaque mouvement respiratoire.

La vésicule commence d̀ se résorber, la goutte graisseuse diminue.

L'intestin s'allonge, sans atteindre. le bord de la proptérygie légèrement incisée.

\section{Alevins de 8 à ro jours.}

Il mesure environ 6 à 6,2 millimètres. Les mâchoires sont formées : In mâchoire supérieure est aussi longue que la mâchoire inférieure et la bouche ne se ferme pas encore complètement. Par transparence sur la face ventrale, on voit les arcs branchiaux. La respiration branchiale est commencée.

La vésicule vitelline est presque complètement résorbée ; le globule graisseux. plus petit est déformé et comme vidé.

Les nageoires pectorales sont toujours les seules nageoires différenciées. mais elles sont très résistantes. Elles sont pigmentées ainsi que la proptérygie par de nombreux mélanophores qui, de ponctués sont devenus f́toilés ; ils sont irréqulièrement répartis ainsi que l'indique le dessin et ne laissent pas encore prévoir l'emplacement des futures nageoires.

D'énormes mélanophores sont étalés sur la vésicule en résorption, grou- pés surtout autour de l'anus.

A chaque myotome correspond un mélanophore en étoile situé sur unc ligne abdominale. Chaque myotome est souligné par un chromatophore allongé en filet qui lui est superposé.

Dimensions :

Longueur totale : 6,2 millimètres ; - Hauteur de la tête : 0,78 millimètres ; - $\operatorname{CEil~:~3,6~4,2~millimètres;~-~Hauteur~maxima~du~tronc:~}$ 
I,2 millimètre; - Longueur du museau à l'anus : 2,75 millimètres; Longueur de l'anus à l'extrémité de la chorde : $3 \mathrm{I}$, I millimètres. — Rapport des deux longueurs : 0,88 .

Les petits alev̄ins étaient conservés dans un aquarium à eau courante. La nourriture qui leur était fournie dès le 4 " jour, était constituée par du plancton vivant, prélevé au filet dans un étang de la Station et distribué chaque jour. L'époque la plus délicate de leur élevage est le moment de la résorption de la vésicule, au moment où ils doivent commencer à s'alimenter eux-mêmes. C'est à ce moment que nos alevins sont morts, âgés de 10 à 12 jours. Ils n ont pas mangé de plancton végétal ni animal. Il semble que d'autres facteurs soient ì mettre en cause pour expliquer l'échec de mon élevage : la composition physico-chimique de l'eau en particulier.

P. Vivier, ayant essayé, en 1939 , d'élever en aquarium à la Station d'Hydrobiologie du Paraclet, de jeunes alevins de Perche issus d'une ponte dont l'éclosion avait parfaitement réussi, n'a pu, lui aussi, les conserver que quelques jours seulement.

Néanmoins, il sera intéressant de tenter de nouveaux essais au printemps prochain en modifiant les conditions d'expériences.

L'élevage des Perches en aquarium et en pisciculture présente donc bien des difficultés et $\boldsymbol{n}$ 'est pas à conseiller. Il faut placer les jeunes Perches dans des conditions aussi voisines que possible des conditions naturelles : bacs largement ouverts, exposés au soleil ou tout au moins bien éclairés. plantes aquatiques, etc..., et la réussite reste incertaine.

\section{BIBLIOGRAPHIE}

Duspiva (F.). - Beitrage zür Physiologie der Melanophoren von Fischembryonen. SitzBer. - Akad. Wiss. Wien, Mathem-nat urw. Klasse, Abt. I, 140. Band, 7 Heft, 1931.

Guenaux (G.). - Pisciculture 1923.

SchindeEr (O.). - Uber die Brut von vier einheimischen Süsswasserfischen (Acsche, Hecht, Flussbarsch und Karpfen). - Allgemeine Fischerei Zeitung, 1934, 59, 20, pp. 305 à 319 .

Smolian (K). - Merkbuch der Binnenfischerei, Berlin 1920.

Spillamann (J.). - Observations sur la Biologie de la Carpe (Cyprinus Carpio L.) et le développement de ses alevins. - Bull. Soc. Centr. d'Aquiculture et de Pêche. T. XLIV, 1937, pp. 86 à 96 et 101 à 113.

Wuntz (A.). - Développement, Biologie et Nutrition des jeunes alevins de Brochets. - Bull. Franf. de Piscicullure, no 135, 1944. 